\title{
Mortality in Kidney Transplantation
}

\author{
Karima Boubaker, Madiha Mahfoudhi*, Amel Gaieb Battikh, Hayet Kaaroud, \\ Ezzeddine Abderrahim, Taieb Ben Abdallah, Adel Kheder
}

Department of Internal Medicine A, Charles Nicolle Hospital, Tunis, Tunisia

Email: ${ }^{*}$ madiha mahfoudhi@yahoo.fr

Received 18 March 2015; accepted 22 May 2015; published 25 May 2015

Copyright (C) 2015 by authors and Scientific Research Publishing Inc.

This work is licensed under the Creative Commons Attribution International License (CC BY).

http://creativecommons.org/licenses/by/4.0/

(c) (i) Open Access

\begin{abstract}
It's a retrospective study whose aim was to evaluate the incidence and the mortality in a population including 329 patients who received first kidney transplants from a living donor in 269 cases and cadaveric donor in 60 cases at Internal Medicine A department between June 1986 and December 2003. Aetiologies of mortality in our kidney transplant recipients were determined. There were 157 males and 75 females having an average age of 30.8 years. After a period of follow-up of 5.64 years, 51 patients $(21.98 \%)$ died. Aetiologies of mortality were multiple and were known in approximately $98 \%$ of cases. Infections were observed in 25 cases. Cancer was observed in 7 cases (13.72\%). Patient survival was not affected by gender, donor age or cause of donor death. Infections represent the major cause of mortality in our patients even many years after kidney transplantation. The maximum of death occurred in the 8th year after kidney transplantation.
\end{abstract}

\section{Keywords}

Kidney Transplantation, Aetiology, Mortality

\section{Introduction}

Kidney transplantation is the treatment of choice for patients with end-stage renal failure. However such patients are increasingly older and have additional co-morbid conditions leading to high mortality rates after transplantation. This poor prognosis of allograft recipients is due to an interaction between high prevalence of classic risk factors and inherent conditions to kidney transplantation [1]. Hence, cardiovascular death rate is higher than in the general population, even after stratifying for age, sex, and race. The aim of this review is to evaluate retrospectively the incidence and aetiologies of mortality in our kidney transplant recipients.

\section{Material and Methods}

The study population included 329 patients who received first kidney transplants from a living donor in 269

\footnotetext{
${ }^{*}$ Corresponding author.
}

How to cite this paper: Boubaker, K., Mahfoudhi, M., Battikh, A.G., Kaaroud, H., Abderrahim, E., Abdallah, T.B. and Kheder, A. (2015) Mortality in Kidney Transplantation. Open Journal of Nephrology, 5, 25-28. 
cases and cadaveric donor in 60 cases at Internal Medicine A department between June 1986 and December 2003. Exclusion criteria were death occurred during kidney transplantation.

We collected all causes of mortality occurred in these patients. We studied age, sex, aetiologies of death and patient survival rates.

All patients received induction immunosuppression during the first few days after receiving the allograft. The induction agent has evolved over the years from Minnesota ALG, to monoclonal antilymphocyte antibodies OKT3 and thymoglobulin. None of the patients had received monoclonal anti-CD25 anti-bodies.

Initiation of cyclosporine treatment post-transplant was delayed until the serum creatinine was $\leq 2.5 \mathrm{mg} / \mathrm{dl}$. All patients were started on cyclosporine microemulsion (Neoral). Prior to 1995, most patients received azathioprine (Imuran) in addition to cyclosporine. However since 1995, all patients received mycophenolate mofetil (Cellcept) instead of azathioprine as part of triple immunosuppression protocol that also included Neoral and prednisone. None of the patients were treated by sirolimus (Rapamycin).

Clinical data were obtained mainly from an electronic database that contains all the clinical and laboratory information in our patients. Age, sex, cause of end-stage renal disease (glomerulonephritis, diabetes, or other causes), pretransplant cardiovascular disease, vascular calcifications, time from first treatment for end-stage renal disease to kidney transplantation and acute tubular necrosis were recorded.

Delayed graft function is defined as the need for dialysis within 1 week after kidney transplantation.

Data in the manuscript are expressed as means \pm standard deviation of the mean unless indicated otherwise. Statistical analysis used STATVIEW logician.

\section{Results}

There were 157 males and 75 females aged mainly of 30.8 years (16 - 61 years).

After a period of follow-up of 5.64 years ( 0 - 17.2 years), 51 patients (21.98\%) died.

The maximum of death occurred in the 8th year after kidney transplantation.

Aetiologies of mortality were multiple and were known in approximately 98\% of cases (Table 1).

Infection were observed in 25 cases (49.01\%): no specific infection in 19 cases, pulmonary aspergillosis in 1case, tuberculosis in 2 cases, listeriosis in 1case, varicelle in 1 case and perforation of CMV intestinal lesions in 1 case. Cancer was observed in 7 cases (13.72\%) with cerebral localisation in 2 cases, hepatic in 1case, gastric in 1case, maxillary in 1case, cutaneous in 1 case and renal lymphoma in 1case. Cirrhosis was observed in 6 cases (11.76\%), pulmonary emboli in 2 cases (3.92\%). Other causes were observed in 11 cases (21.56 \%).

The 1-, 5-, 10- and 20-year patient survival rates were $99.60 \%, 91.20 \%, 80.60 \%$ and $62.70 \%$ respectively (Figure 1). Patient survival was not affected by gender, donor age or cause of donor death.

\section{Discussion}

Since the initial successful kidney transplantation in humans, the field of kidney transplantation has made significant progress.

Patient survival and graft survival have improved tremendously. High comorbidity is associated with an increased risk for patient death but not for graft failure or acute cellular rejection, both in the perioperative period and after first 3 months of kidney transplantation [2].

Indeed, based on age and number of co morbid conditions, kidney transplant recipients have been successfully classified to have low, medium, or high risk of death [3] [4].

The most common comorbid conditions found in literature were posttransplant diabetes mellitus diabetes (30\%) and heart failure (12\%) [2] [5]. The occurrence of fatal and nonfatal cardiovascular events after successful kidney transplantation not only relates to baseline cardiovascular risk factors present at transplantation, but also to immunosuppressive drugs and post transplantation traditional and non-traditional risk factors. Posttransplantation anemia is an independent risk factor for cardiovascular morbidity and mortality in kidney transplant recipients. There are multiple causes of Posttransplantation anemia such as impaired kidney function, iron deficiency, medications, infections, acute rejection, inflammation, and erythropoietin deficiency.

Current guidelines recommend evaluation for hemoglobin level of less than $12 \mathrm{~g} / \mathrm{dL}$ and treatment when the value falls less than $11 \mathrm{~g} / \mathrm{dL}$ and a target of 11 to $12 \mathrm{~g} / \mathrm{dL}$. Additional treatments may entail removing the cause of the anemia, nutritional supplementation, and/or an erythrocyte stimulating agent [6]. In our study, we found 2 


\section{Survival Rate}

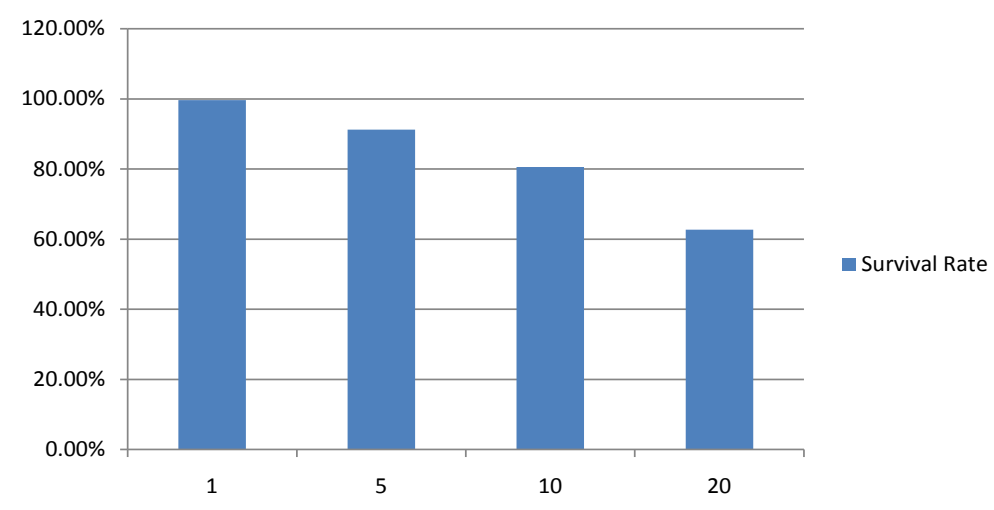

Figure 1. Survival rate histogram.

Table 1. Aetiologies of mortality in our series.

\begin{tabular}{cc}
\hline Aetiologies of mortality & Number of patients \\
\hline Infections & 25 \\
Cancers & 7 \\
Cirrhosis & 6 \\
Pulmonary emboli & 2 \\
Other causes & 11 \\
\hline
\end{tabular}

cases of pulmonary emboli.

Other important risk factors of co morbidity are immunosuppressive regimen, metabolic disorders and infections [2]. Infection-related mortality was observed in $49.01 \%$ of cases in our study. They were specific infection in almost the half of the cases. Indeed, in the last decade, infection-related mortality among kidney transplant recipients has not decreased. Although better control of invasive viral infections has been achieved, bacterial and fungal invasive infections remain important causes of mortality in this population. Cytomegalovirus infection in kidney transplant recipients causes decreased patient survival. It was a case of death in one of our patients.

Early targeted treatment interventions groups at high risk for poor outcomes may be indicated and, consequently, help minimize mortality [2].

In our series, the maximum of death occurred in the 8th year after kidney transplantation. This may be due to either elevated frequency of infections caused by prolonged immunosuppression or high carcinogenic risk of the prescribed treatment.

Twelve variables independently predicted death: age, race, cause of kidney failure, body mass index, comorbid disease, smoking, employment status, serum albumin level, year of first renal replacement therapy, kidney transplantation, time to transplant wait-listing and time on the wait list [7].

Increasing donor age (but not recipient age), recipient diabetes, and grafts from adult offspring were independently associated with poorer patient survival in the first 3 years after transplantation [8]. Poorer graft survival was independently associated with donor age older than 59 years, and female recipients [8].

Cancer related mortality is observed in $13.72 \%$ of cases in our study. In fact, immunosuppression in solid organ transplant recipients is associated with increased risk of a broad range of cancers. Increased cancer risk mainly for Kaposi's sarcoma, non-Hodgkin's lymphoma, melanoma, and lip cancer, is rapidly reversible on reduction or cessation of immunosuppression regimen [9].

Our 1-, 5-, 10- and 20-year patient survival rates were 99.60\%, 91.20\%, 80.60\% and 62.70\% respectively, were better than those recently published with non-extended criteria donors [10] [11].

In the quoted study, however, a decidedly inferior graft survival was seen among extended criteria donor organs, $87.4 \%$ and $66.4 \%$ at 1 and 5 years, respectively [10]. In fact expanded criteria deceased kidneys render a 
survival benefit, but should be given principally to patients over the age of 40 years and when the waiting list is long.

Patient survival is reduced among recipients aged $>50$ years compared with those $<50$ years of age and in female-to male donations [10].

\section{Conclusion}

Infections represent the major cause of mortality even many years after kidney transplantation. Cancer origin should also be considered in kidney transplantation mortality.

\section{Conflict of Interests}

The authors declare no conflict of interests.

\section{References}

[1] Sarnak, M.J., Levey, A.S., Schoolwerth, A.C., Coresh, J., Culleton, B., Hamm, L.L., et al. (2003) Kidney Disease as a Risk Factor for Development of Cardiovascular Disease: A Statement from the American Heart Association Councils on Kidney in Cardiovascular Disease, High Blood Pressure Research, Clinical Cardiology, and Epidemiology and Prevention. Circulation, 108, 2154-2169. http://dx.doi.org/10.1161/01.CIR.0000095676.90936.80

[2] Hernández, D., Rufinoa, M., González-Posadaa, J.M., Estupiñán, S., Pérez, G., Marrero-Miranda, D., et al. (2008) Predicting Delayed Graft Function and Mortality in Kidney Transplantation. Transplantation Reviews, 22, 21-26. http://dx.doi.org/10.1016/j.trre.2007.09.007

[3] Wright, L.F. (1991) Survival in Patients with End-Stage Renal Disease. American Journal of Kidney Diseases, 17, 2528. http://dx.doi.org/10.1016/S0272-6386(12)80245-9

[4] Miskulin, D.C., Meyer, K.B., Martin, A.A., Fink, N.E., Coresh, J., Powe, N.R., et al. (2003) Comorbidity and Its Change Predict Survival in Incident Dialysis Patients. American Journal of Kidney Diseases, 41, 149-161. http://dx.doi.org/10.1053/ajkd.2003.50034

[5] Gnatta, D., Keitel, E., Heineck, I., Cardoso, B.D., Rodrigues, A.P., Michel, K., et al. (2010) Use of Tacrolimus and the Development of Posttransplant Diabetes Mellitus: A Brazilian Single-Center, Observational Study. Transplantation Proceedings, 42, 475-478. http://dx.doi.org/10.1016/j.transproceed.2010.02.021

[6] Blosser, C.D. and Bloom, R.D. (2010) Post Transplant Anemia in Solid Organ Recipients. Transplantation Reviews, 24, 89-98. http://dx.doi.org/10.1016/j.trre.2010.01.006

[7] Van Walraven, C., Austin, P.C. and Knoll, G. (2010) Predicting Potential Survival Benefit of Renal Transplantation in Patients with Chronic Kidney Disease. CMAJ, 182, 666-672. http://dx.doi.org/10.1503/cmaj.091661

[8] Fuggle, S.V., Allen, J.E., Johnson, R.J., Collett, D., Mason, P.D., Dudley, C., et al. (2010) Factors Affecting Graft and Patient Survival after Live Donor Kidney Transplantation in the UK. Transplantation, 89, 694-701. http://dx.doi.org/10.1097/TP.0b013e3181c7dc99

[9] van Leeuwen, M.T., Webster, A.C., McCredie, M.R., Stewart, J.H., McDonald, S.P., Amin, J., et al. (2010) Effect of Reduced Immunosuppression after Kidney Transplant Failure on Risk of Cancer: Population Based Retrospective Cohort Study. BMJ, 340, c570. http://dx.doi.org/10.1136/bmj.c570

[10] Shaheen, M.F., Shaheen, F.A., Attar, B., Elamin, K., Al Hayyan, H. and Al Sayyari, A. (2010) Impact of Recipient and Donor Nonimmunologic Factors on the Outcome of Deceased Donor Kidney Transplantation. Transplantation Proceedings, 42, 273-276. http://dx.doi.org/10.1016/j.transproceed.2009.12.052

[11] Merion, R.M., Ashby, V.P., Robert, M.A., et al. (2005) Deceased-Donor Characteristics and the Survival Benefit of Kidney Transplantation. JAMA, 294, 2726. http://dx.doi.org/10.1001/jama.294.21.2726 